\title{
Clinical Evaluation in Isoflurane and Sevoflurane Anesthesia in Rat
}

\author{
Flavia RUXANDA ${ }^{1}$, Lucia BEL ${ }^{1}$, Cristian RAŢIU ${ }^{2 *}$, Viorel MICLĂUȘ ${ }^{1}$, Cosmin PEȘTEAN $^{1}$, Liviu OANA ${ }^{1}$ \\ ${ }^{1}$ Faculty of Veterinary Medicine, University of Agricultural Sciences and Veterinary Medicine Cluj- \\ Napoca, Romania. \\ ${ }^{2}$ Faculty of Medicine and Pharmacy, University of Oradea, Romania \\ *corresponding author: ratiu_cristian@yahoo.com
}

Bulletin UASVM Veterinary Medicine 73(2)/2016

Print ISSN 1843-5270; Electronic ISSN 1843-5378

doi:10.15835/buasvmen-vm:12030

\begin{abstract}
The present study focused on evaluation of induction, maintenance and emergence of inhaled anesthesia and comparison of these anesthetic times for isoflurane and sevoflurane. The biological material consisted of 12 Wistar rats divided into 2 groups, each containing a number of 6 rats. One group was anesthetized with isoflurane and the other with sevoflurane. We monitorized the rats during all stages of anesthesia, observing how the induction established, the state of the rats throughout the anestesia and the moment of the first recovery signs, the moment the rats became active as well as the moment of full recovery from anesthesia. Both isoflurane and sevoflurane rapidly induced the anesthesia, without excitation signs and the emergence was significantly faster in sevoflurane group in comparison to the isoflurane group, being accompanied by horripilation in both anesthetics.
\end{abstract}

Keywords: anesthesia, isoflurane, sevoflurane.

\section{INTRODUCTION}

There is a permanent concern in utilizing less and less invasive surgical techniques, accompanied, as much as possible, by a more rapid recovery from anesthesia.

For most of the anesthetics, especially for the inhaled ones, the recovery time and the manner it unfolds are of major importance. Introduction of less soluble inhaled anesthetics decreased the emergence time.

Beside the fast recovery from anesthesia, the side effects that can emerge under the action of anesthetics are particularly important. Among the minor side effects which can appear after inhaled anesthetics we mention: emesis, vomiturition, dizziness, drowsiness, headaches, shakiness or cough, some of them being difficult to quantify in animals.

Most of the studies in specialty literature are centred on clinical aspects encountered in inhaled anesthesia in humans (Elcock and Sweeney, 2002;
Huddy, 2010; Jindal et al., 2011), while studies on animals are limited. Inhaled anesthetics were avoided for a long time in anesthesia induction because of their pungent odour.

The recovery times reported in human patients are shorter after sevoflurane utilization, in comparison to isoflurane, but there are inconsistencies between the reported results. Some authors claim they did not observe any difference between the two anesthetic agents (Quinn et al., 1994), while others stated that the recovery was faster after isoflurane anesthesia in comparison to sevoflurane (Chiu et al., 2000).

In order to assess the clinical aspects that accompany anesthesia with inhalation substances, we observed 6 animals anesthetized with isoflurane and 6 with sevoflurane, subsequently comparing the registered times, from a statistically point of view.

The proposed objectives were observation of induction, maintenanceand emergence afterinhaled 
anesthesia and comparison of the anesthetics times between isoflurane and sevoflurane.

\section{MATERIAL AND METHODS}

The biologic material was represented by 12 Wistar female rats, 6 weeks old, who came from the biobase of University of Medicine and Pharmacy "Iuliu Hatieganu”, in Cluj-Napoca.

The experimental protocol was approved by the Bioethics Committee of University of Agricultural Sciences and Veterinary Medicine in Cluj-Napoca and took place in Department of Anesthesiology and Surgical Propedeutics and the animals were brought to UASVM's biobase one week prior to the initiation of anesthesia.

Animals were divided in 2 groups: one anesthetized with isoflurane $(n=6)$ and the other with sevoflurane $(n=6)$. In order to attain a uniform anesthesia, we maintained a concentration of $1.5 \%$ anesthetic and $11 \mathrm{O}_{2}$ /minute, for 2 hours long in the case of isoflurane, $2 \%$ anesthetic and $11 \mathrm{O}_{2}$ /minute, for 2 hours long for sevoflurane, respectively. Based on the the data in the specialty literature, at these doses, we obtain a minimum alveolar concentration (MAC) of approximately 1 for both isoflurane (Mazze et al., 1985) and sevoflurane (Tamada et al., 1986).

After the anesthesia, we registered the moment when the first signs of recovery appeared, the moment when the rats became active, as well as the moment of full recovery from anesthesia.

In order to register the moment of the first recovery signs, we measured the time immediately after closing of the vaporizer until the movement of the limbs, lips, whiskers, opening of the palpebral fissure etc.

The moment when the rats were catalogued as active was when they presented the redress reflex (attempt to attain a patruped position) for the

Tab. 1. Recovery times for isoflurane anesthesia first time, and in order to register the time passed until the complete recovery from anesthesia, we recorded the moment when the rats adopted a patruped position, were alert (dynamic) and started grooming and cleaning themselves.

For the statistical analysis we used the GraphPad Software, where we applied the Student's t-test.

\section{RESULTS AND DISCUSSIONS}

Induction of isoflurane and sevoflurane anesthesia took place without excitation signs. Rats did not present apnea or cough upon inhalation of anesthetics, and the anesthesia installed in 1-2 minutes after airtightening the induction chamber and starting the vaporizer.

Throughout the whole sevoflurane anesthesia, a rat presented disquiet signs with short periods of calmness.

The recovery times from isoflurane and sevoflurane anesthesia are presented in Tab. 1. and Tab. 2., respectively. The average times registered for the first recovery signs of the animals were $6 \pm 2.1$ minutes in the case of isoflurane and 3.5 \pm 1.8 minutes for sevoflurane. Recovery of the animals took place $8.5 \pm 2.2$ minutes after closing the vaporizer in the case of isoflurane and $5.6 \pm$ 2.4 minutes for sevoflurane, and the average time to full recovery was $12 \pm 3$ minutes for isoflurane and $7.8 \pm 3$ minutes in rats anesthetized with sevoflurane.

Standard deviations of the recovery times after isoflurane and sevoflurane anesthesia are presented in Tab. 3. Upon statistical analysis we compared the obtained values for the first recovery signs, activity and full recovery of rats anethetized with isoflurane with the ones from rats from sevoflurane group.

The difference between the registered times in the two groups was not statistically significant

\begin{tabular}{ccc}
\hline & Isoflurane & \\
\hline First recovery signs & Recovery (activity) & Full recovery \\
\hline 3 minutes & 5 minutes & 8 minutes \\
\hline 4 minutes & 7 minutes & 10 minutes \\
\hline 9 minutes & 10 minutes & 15 minutes \\
\hline 7 minutes & 10 minutes & 14 minutes \\
\hline 7 minutes & 11 minutes & 15 minutes \\
\hline 6 minutes & 8 minutes & 10 minutes
\end{tabular}


Tab. 2. Recovery times for sevoflurane anesthesia

\begin{tabular}{ccc}
\hline & Sevoflurane & \\
\hline First recovery signs & Recovery (activity) & Full recovery \\
\hline 3 minutes & 7 minutes & 10 minutes \\
\hline 5 minutes & 8 minutes & 10 minutes \\
\hline 2 minutes & 4 minutes & 7 minutes \\
\hline 1 minute & 2 minutes & 3 minutes \\
\hline 6 minutes & 8 minutes & 11 minutes \\
\hline 4 minutes & 5 minutes & 6 minutes \\
\hline
\end{tabular}

Tab. 3. Standard deviations of the recovery times

\begin{tabular}{|c|c|c|c|c|c|}
\hline \multicolumn{3}{|c|}{ Isoflurane } & \multicolumn{3}{|c|}{ Sevoflurane } \\
\hline First recovery signs & Recovery & Full recovery & First recovery signs & Recovery & Full recovery \\
\hline $2.19089^{\mathrm{NS}}$ & $2.25831^{\mathrm{NS}}$ & $3.03315^{*}$ & $1.870829^{\mathrm{NS}}$ & $2.42212^{\mathrm{NS}}$ & $3.060501^{*}$ \\
\hline
\end{tabular}

NS - not statistically significant, ${ }^{*}$ - satistically significant $(\mathrm{p}<0.05)$.

in the case of first recovery signs or activity of the animals, but was statistically significant in the case of full recovery $(\mathrm{p}<0.05)$.

Thus, the emergence from anesthesia was faster after sevoflurane administration in comparison to isoflurane.

Recovery from anesthesia took place gradually, witouth excitation signs. No animals presented clinical signs that would suggest irritation of the respiratory airways (cough, nasal discharges) after anesthesia. On the other hand, most of the rats presented horripilation during recovery.

In the specialty literature there are studies concerning the time and aspects of recovery from anesthesia, but most of them were retrospective and carried out on human subjects. There are studies that also focused on animals, the recovery times being important because a lot of clinics do not possess spaces for accommodating the patients after inverventions, the owners being obligated to take the animal home as soon as possible. In these cases, the necessary emegence time from an anesthesia has a major importance. Also, the aspects that accompany the recovery from anesthesia are important because an excitation state cand lead to certain complications (e.g. wound dehiscence).

In studies carried out on dogs, the researchers compared the time and characteristics of recovery from anesthesia after administration of various inhaled agents: sevoflurane, isoflurane and halothane. Dogs recovered calmly, without excitation signs after sevoflurane anesthesia and were able to stand up 10 minutes earlier in comparison to those anesthetized with isoflurane and 85 minutes earlier in comparison to the ones in which halothane was utilized (Taske et al., 1998). The situation reported by these authors resembles the one we encountered in our study carried out on rats: faster recovery after sevoflurane anesthesia in comparison to isoflurane and the absence of excitation in the case of both anesthetics. This situation is justified regarding the fact that sevoflurane has a lower blood-gas partition coefficient than isoflurane.

Other studies concentrated on the frequency and severity of cough upon recovery from isoflurane anesthesia and the influence smoking has on coughing extent and respiratory function (Eun and Bishop, 1998). From a total of 68 human patients, 52 coughed during emergence from isoflurane anesthesia. Cough incidence was the same in smokers in comparison to non-smokers. Most of the patients cough when recovering from general anesthesia attained with and endotracheal tube.

Smooth recovery from anesthesia is desirable because cough can become a problem after ocular and neurosurgical interventions. In isolated cases, strong cough can even tear open an abdominal 
suture. In our study, rats did not present cough when recovering from anesthesia, but the animals were not intubated. Obviously, in animals we can not talk about smoking, although researches tracked down changes in the respiratory tract of pets with smoking owners (Cătoi, 2006).

Comparative studies were also carried out, concerning the inhalatory induction with sevoflurane and the intravenous one with propofol. For a long time, clinicians avoided inhalatory induction, fearing the potential excitation and respiratory simptoms induced by the pungent smell of available volatile anesthetics. Nevertheless, sevoflurane has certain advantages: less pungent that isoflurane, lower blood-gas solubility, which make it a better candidate for anesthesia induction. Upon comparison of inhalational anesthesia with sevoflurane to intravenous one with propofol, results have shown that there are differences between recovery times after the two types of anesthesia (Beverly et al., 1999).

Other studies concentrated on postoperative recovery and complications after propofol, isoflurane, sevoflurane and desflurane anesthesia. There were no noticeable differences between the recovery from propofol and isoflurane anesthesias. However, in humans, recovery was faster after sevoflurane in comparison to isoflurane. A minor difference was encountered in the full recovery time after sevoflurane and isoflurane ( 5 minutes), but not in the case of other anesthetics. Also, in our study, rats recovered approximately 4 minutes earlier after sevoflurane anesthesia in comparison to isoflurane. The recovery times presented small differences, but were in the favor of sevoflurane. Patients anesthetized with isoflurane presented a significantly more frequent postoperative drowsiness in comparison to sevoflurane, but there were no other significant differences regarding the postoperative complications (Gupta et al., 2004). In rats, we did not observe drowsiness, moreover, they started grooming themselves immediately after full recovery from anesthesia. Other articles contain information on the emergence times after sevoflurane and isoflurane anesthesia, and mention that sevoflurane would have an advantage in comparison to isoflurane from this point of view and also that it has less of an influence on cardiac function (Frink et al., 1992). The study of TerRiet et al. (2000) concentrated on the irritability of anesthetic agents, their results suggesting that sevoflurane has a smaller irritability degree than isoflurane $(p<0.05)$ at a 2 CAM concentration.

Some others mention that the recovery time from sevoflurane anesthesia is $7.5 \pm 0.5$ minutes, as for isoflurane $18.6 \pm 2.0$ minutes (Frink et al., 1992), while others report 7 minutes in the case of sevoflurane and 11.5 minutes for isoflurane (Wiesner et al., 1994). Although the reported results are from human patients, they are not very different from what we registered for rats anesthetized with sevoflurane and isoflurane (7.8 \pm 3 minutes for sevoflurane and $12 \pm 3$ minutes for isoflurane until full recovery of animals).

Recovery from anesthesia was faster after sevoflurane anesthesia in comparison to isoflurane in the study carried out by Smith et al. (1992) in humans. Thus, the recovery time after isoflurane anesthesia was $6.7 \pm 2.2$ minutes, while after sevoflurane it was $4.0 \pm 2.0$ minutes. The registered time was significantly longer after isoflurane, similar to what we observed in our study.

Other authors also report that there are significant differences between the recovery times after isoflurane and sevoflurane anesthesia, in the favor of sevoflurane (Robinson et al., 1999). As we mentioned, this is logical, given the fact that sevoflurane presents a lower blood-gas partition coefficient.

The recovery times after anesthesia were mostly registered in human patients, less in animals, but most of them show that recovery is faster after sevoflurane anesthesia than the one with isoflurane, also sustained by the fact that sevoflurane has a lower solubility in blood. There are studies that sustain that recovery was faster after isoflurane (time to opening the palpebral fissure: $6.8 \pm 2.2$ minutes isoflurane and $10.7 \pm 4.4$ minutes sevoflurane) (Chiu et al., 2000) and studies in which authors did not observe any difference between the recovery times after isoflurane and sevoflurane anesthesia (Quinn et al., 1994).

There are authors who affirm that recovery from anesthesia is faster in the case of women in comparison to men, stating that is uncertain if this fact is due to sex or differences in the administration of anesthetics or type of surgery. However, the authors write that this might be due to the different quantity of administered anesthetic (smaller doses in women), but in spite of administering similar doses, it seems like women are actually less susceptible to the hypnotic effect 
of anesthetics than men. Although the study was carried out on humans, same mechanisms could also be involved in the emergence from anesthesia in animals. The authors write that the differences between the recovery times from anesthesia are more pronounced in younger patients, which suggests a hormonal influence. It was shown that progesterone increases the potency of inhalational anesthetics and induces sleep in humans and probably in animals as well (Buchanan et al., 2006). Our study only included female rats, thus we can not say if sex had any influence on the recovery times from anesthesia, but the fact that they were short is a certainty.

The rapid recovery from anesthesia is also desirable when performing field interventions and also in wild animals. The two anesthetics were even successfully used in exotic animals. In other authors' opinion, isoflurane was not sufficiently assessed in wild animals. It appears like the duration of induction and emergence from anesthesia depend on the season in the case of squirrels, when anesthesia is performed in the field. This fact was not noticed in Allegheny woodrats, the recovery times being similar in summer, as well as winter (Parker et al., 2008).

Induction of sevoflurane anesthesia with a mask was faster and had a better quality than the one with isoflurane in adult dogs in the study carried out by Johnson and et al. (1998). Sevoflurane is better tolerated during mask induction because it is less pungent and has a decreased irritability. Sevoflurane is less potent than isoflurane, thus the vaporizer has to be set on a higher concentration than isoflurane. Although the quantity of sevoflurane metabolized by the liver is slightly larger than in the case of isoflurane, the metabolic rate is similar for the two anesthetics because of sevoflurane's insolubility (hence its rapid elimination).

The low solubility of sevoflurane in blood (0.69) suggests that recovery from anesthesia should be faster than the one from isoflurane (1.4). The prediction proved to be valid in a rat study. Recovery of the muscle coordination after anesthesia with 1.2 MAC for 2 hours long required $14.2 \pm 8.1$ minutes for sevoflurane, 23.4 \pm 7.6 minutes for isoflurane (Eger et al., 1987). We anesthetized the rats for 2 hours long and the full recovery, including muscle coordination took place faster: $7.8 \pm 3$ minutes for sevoflurane and
$12 \pm 3$ minutes for isoflurane, which is justified, given the fact that we administered 1 MAC in the case of both anesthetics. Our recorded times are approximately half of the ones reported in the study carried out by Eger et al. (1987).

Our study included 6 animals/group because of the capacity of the induction box, but for stronger statistical analysis results, it would be reccommended to use larger anesthesia chambers. Further studies which monitor other parameters such as: heart rate, repiration rate etc. in animals are necessary, since in humans it seems that sevoflurane induces a higher incidence of complications (Elcock and Sweeney, 2002). Also, studies assessing the clinical aspects of inhaled anesthesia on rats with different ages could reveal differences among younger and older subjects. Studies conducted in humans mention that young subjects display agitation upon emergence after sevoflurane and desflurane anesthesia (Huddy, 2010).

\section{CONCLUSIONS}

Induction of anesthesia with isoflurane and sevoflurane in rats was rapid, without excitation signs. Recovery from anesthesia took place faster after sevoflurane administration in comparison to isoflurane, being accompanied by horripilation in the case of both anesthetics taken into study.

\section{REFERENCES}

1. Beverly P, Lombard L, Roaf E, Drager L, Calalang I, Philip J (1999). Comparison of Vital Capacity Induction with Sevoflurane to Intravenous Induction with Propofol for Adult Ambulatory Anesthesia. Anesthesia \& Analgesia 89(3):623.

2. Buchanan FF, Myles P, Leslie K, Forbes A, Ciucuttini F (2006). Gender and recovery after general anesthesia combined with neuromuscular blocking drugs. Anesth Analg 102:291-307.

3. Cătoi C (2006). Anatomie patologică specială, vol. 1, ed. AcademicPres, Cluj-Napoca.

4. Chiu CL, Chan YK, Ong GSY, Dlilkan AE (2000). A comparison of the maintenance and recovery characteristic of sevoflurane-nitrous oxide against isoflurane-nitrous oxide anesthesia. Singapore Med J 41(11):530-533.

5. Eger EI II, Johnson BH (1987). Rates of awakening from anesthesia with I-653, halothane, isoflurane, and sevoflurane: A test of the effect of anesthetic concentration and duration in rats. Anesth Analg 66:977-982.

6. Elcock DH, Sweeney BP (2002), Sevoflurane vs. isoflurane: a clinical comparison in day surgery, Anaesthesia 57(1):53-57. 
7. Eun K, Bishop M (1998). Cough During Emergence from Isoflurane Anesthesia. Anesthesia \& Analgesia 87(5):1170-1174.

8. Frink EJ, Malan TP, Atlas M, Dominiquez LM, Dinardo JA Brown BR (1992). Clinical comparison of sevoflurane and isoflurane in healthy patients. Anesth Analg 74(2):241245.

9. Gupta A, Stierer T, Zuckerman R, Sakima N, Parker $S$, Fleisher L (2004). Comparison of recovery profile after ambulatory anesthesia with propofol, isoflurane, sevoflurane and desflurane: a systematic review. Anesthesia \& Analgesia 98(3):632-641.

10. Huddy N (2010), Emergence agitation in children, Br. J. Anaesth. 105(1):95-96.

11. Jindal R, Kumra VP, Narani KK, Sood J (2011), Comparison of maintenance and emergence characteristics after desflurane or sevoflurane in outpatient anaesthesia, Indian Journal of Anaesthesia 55(1):36-42.

12. Johnson RA, Striler E, Sawyer D, Brunson D (1998). Comparison of isoflurane with sevoflurane for anesthesia induction and recovery in adult dogs. AJVR 59:478-481.

13. Mazze RI, Rice SA, Baden JM (1985). Halothane, isoflurane and enflurane MAC in pregnant and nonpregnant female and male mice and rats. Anesthesiology 62:339-341.

14. Parker WT, Muller LI, Gerhardt RR, O'Rourke DP, Ramsay EC (2008). Field use of isoflurane for safe squirrel and woodrat anesthesia. Journal of wildlife management 72(5):1262-1266.
15. Quinn AC, Newman PJ, Hall GM, Grounds RM (1994). Sevoflurane anaesthesia for major intra-abdominal surgery. Anaesthesia 49:567-571.

16. Robinson BJ, Uhrich TD, Ebert TJ (1999). A revies of recovery from sevoflurane anaesthesia: comparisons with isoflurane and propofol including meta-analysis. Acta Anaesthesiol Scand 43(2):185-190.

17. Smith I, Ding Y, White PF (1992). Comparison of induction, maintenance, and recovery characteristics of sevoflurane$\mathrm{N} 20$ and propofol-sevoflurane-N2O with propofolisoflurane-N20 anesthesia. Anesth Analg 74(2):253-259.

18. Tamada M, Inoue T, Watanabe Y, Kawakubo Y, Ogoh M, Okumura N, Tamura T, Satoh N (1986). MAC values of sevoflurane. Prog Med 6:3248-3253. (In Japanese with English abstract)

19. Taske S, Xiong S, Schimke E (1998). Sevoflurane (SEVOrane) as an inhalation anesthetic in dogs in comparison with halothane and isoflurane. Tierarzti Prax Ausg K Klien Heim 26:369-377.

20. Terriet MF, Desouza GJ, Jacobs JS, Young D, Lewis MC, Herrington C, Gold MI (2000). Which is most pungent: isoflurane, sevoflurane or desflurane? Brazilian Journal of Anaesthesiology 85:305-307.

21. Wiesner G, Schwurzer S, Horauf K, Hobbhahn J (1994). Emergence times, hemodynamics and adverse effects of sevoflurane and isoflurane: an open, randomized, comparative phase iii study [Article in German]. Anaesthesist 43(9):587-593. 